\title{
Study on Health Management and Service Model of Chifeng Class 3A General Public Hospital
}

\author{
Rong Yu* \\ Urology Surgery, The First Affiliated Hospital of Chifeng University, Chifeng 024000, Inner Mongolia Autonomous Region, \\ China
}

*Corresponding author: Rong Yu, ahdc@163.com

\begin{abstract}
This study investigates the construction of a health management service mode based on large-scale comprehensive hospitals by analyzing health management service modes at home and abroad, that is, taking the Affiliated Hospital of Chifeng University as an example, establish a health examination hospital, undertake core tasks such as health service operation process, system, monitoring, and system formulation, and take the best medical resources. Attract social medical resources to collaborate and give health service mode of health status monitoring, evaluation, and intervention to health service objects, aided by advanced medical technology. To extend the traditional health management service with the hospital as the protagonist to health management with participation from family and society beyond the hospital, forming a closed-loop, rapid, and efficient health service management system. Thus, through graded quality services, to lessen the difficulties of patients seeing a doctor and to solve the problem of overcrowding and minor ailments in major hospitals.
\end{abstract}

Keywords: Health management; Health examination hospital; Class 3A hospitals

Publication date: November 2021; Online publication: November 30, 2021

\section{Introduction}

Health management includes the entire process of continuously monitoring, analyzing, evaluating, and predicting the health risk factors of individuals or groups, including healthy people, sub-health people, and disease people, as well as providing health consultation, guidance, and intervention, in order to achieve the best disease prevention and health maintenance results. In the 1960s and 1970s, the concept and practice of health management were first introduced in the United States. It has seen the application and growth of insurance, occupational protection, and other sectors and fields in the United States, Germany, and Finland. People in the Chifeng area have a strong sense of protection for their own health status and disease prevention in recent years, as their living standards have improved. Furthermore, variables such as population ageing and chronic diseases encourage the rapid growth of health management as a business. Community health centers, medical institutes, and physical examination centers are among the current service modes. The health-care management industry, on the other hand, is still in a disorganized and lowlevel state. It is critical to speed the directional shift and build a new health management service model that is appropriate for Chifeng City and its environs ${ }^{[1]}$.

By examining the development of the existing health management service model in Chifeng City, as well as the opportunities presented by the health management service model, as well as the national positioning of large-scale general hospitals, utilizing existing medical and social resources, ensuring the provision of sustainable and diversified diagnosis and treatment services, and utilizing new forces in the health management industry to explore and reap the benefits In Chifeng, the Affiliated Hospital of Chifeng University is used as the engine of health management service, and the links between health and medical 
services are organically coordinated to change the current situation of health management service being an isolated island and to realize the integrity and continuity of health management service (Figure 1).

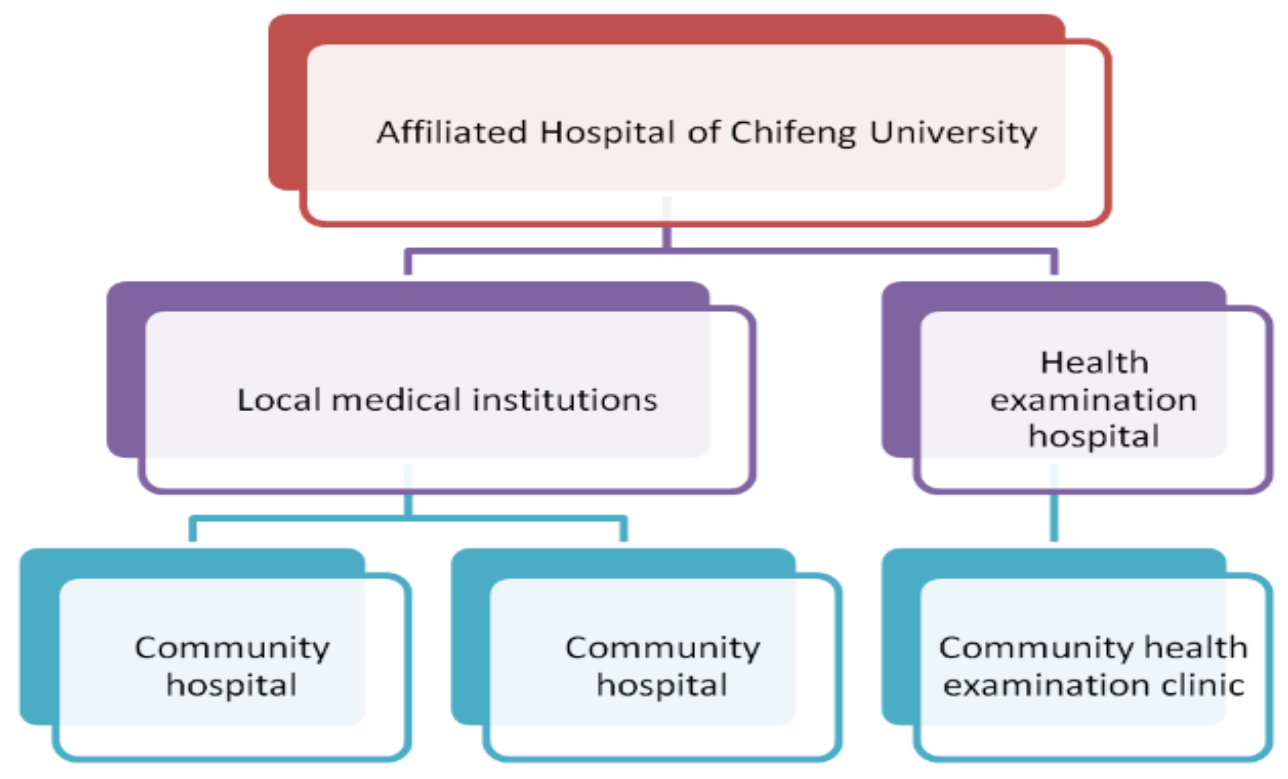

Figure 1. Overall framework of health management service model of Affiliated Hospital of Chifeng University

\section{Establish a health examination hospital in the Affiliated Hospital of Chifeng University}

Large general hospitals serve as a driving force behind health-care management services. To begin, the system must establish independent health examination hospitals. The partnership between health examination hospitals and major general hospitals is a subsidiary (or holding) relationship that operates in a market-oriented manner. To compensate for the shortcomings of traditional health service institutions, large general hospitals must provide full medical resources, technical support, and policy mechanism support to health operation institutions; health examination hospitals are closely linked with hospitals to receive better medical resource support ${ }^{[2-3]}$.

To begin, create a coordinating body for large general hospitals' health management to coordinate the operations of health examination hospitals. Second, we should designate a certain location as the health examination hospital's workplace to ensure that the work is completed. Finally, the personnel mix of health examination hospitals should be set to assure their operation. The health examination hospital has a customer service department for health care, a remote monitoring department, a technical service department, and other business departments that all work together to accomplish their tasks in line with the system. The health care customer service department is made up of a group of health managers who are responsible for overseeing the entire process of health information collection, evaluation, guidance, and promotion of health services; the remote monitoring department provides integrated guidance on the technology of social resources and health services through real-time monitoring; and the health care customer service department is made up of a group of health managers who are responsible for overseeing the entire process of health information collection, evaluation, guidance, and promotion of health services. The technical service department shall provide and maintain a unified health information service platform. The management of the health examination hospital is responsible for establishing the operation system and operation mechanism of the health service operation management system, and attracting and developing health service member units. 


\subsection{Establishment of health service operation system}

The health examination hospital is responsible for establishing the health service operation system and implementing integrated management in terms of technology and service specifications. To attract social resources to participate in the health service system, we must follow the system to implement the service. The system includes: management system, service system, service object satisfaction survey system, supervision system, interest distribution system, etc.

\subsection{Establishment of health service operation mechanism}

The health examination hospital is responsible for formulating the operation mechanism, implementing integrated management in the health management standards and health management processes, and the participation of social resources in the health service system must comply with the operation mechanism. Including incentive mechanism, communication mechanism, cooperation mechanism, etc. For example, how can the health service platform complete service support such as information transmission and followup tracking? The health service object shall consult the health manager. If the health manager cannot solve it, he shall request the superior health manager for consultation and communication by sending a request according to the requirements of the operation mechanism ${ }^{[4]}$.

\subsection{Develop health service operation member units}

Health examination hospitals develop health service operation member units, that is, attract social resources to join the health service operation system and provide health management services. Social resource areas can be local or cross regional. First, attract the existing health examination institutions to join the health service operation system to provide health management services; Secondly, develop community health examination institutions to provide health management services with the help of community houses, equipment, doctors and other resources.

\section{Health management service model and operation mechanism of Affiliated Hospital of Chifeng University}

\subsection{Affiliated Hospital and health examination hospital of Chifeng University}

The Affiliated Hospital of Chifeng University, as the medical service support organization of the health examination hospital, has a corresponding supporting management mechanism in terms of medical resources and systems. Large comprehensive hospitals provide good policy support for physical examination hospitals in the system to make up for the shortage of resources of traditional health service institutions. At the same time, physical examination hospitals are closely connected with hospitals, so as to get better support, such as two-way referral support provided by both parties ${ }^{[5]}$.

\subsection{Other medical institutions and health examination hospitals}

Based on the health service model of the Affiliated Hospital of Chifeng University, it is bound to attract other medical institutions to join the health service system. In terms of health services, local medical institutions need to provide support to health service institutions. If patients are hospitalized in medical institutions, they will carry out services according to the existing medical service model of the hospital; If the patient is discharged from the hospital, the local health service institution shall provide follow-up health services and health tracking for the patient; In case of emergency, the local health institution shall provide daily health monitoring information for the hospital after admission.

\subsection{Community health center and health examination hospitals}

Health examination hospitals implement integrated management in terms of system, mechanism, 
technology and service specification, and health service operation institutions issue systems to attract social resources to participate and follow the system requirements; According to the system, the health examination hospital provides the community with health service platform, information transmission, follow-up tracking and other service support, and implements service supervision on the community.

\subsection{Social medical service resources and Affiliated Hospital of Chifeng University}

Based on the health management service model of the Affiliated Hospital of Chifeng University, it is bound to attract local health examination institutions to join the health service system, such as private physical examination institutions. These physical examination institutions provide daily health management services for health service objects. Once these physical examination institutions cannot provide services for health service objects, health service objects need to go to local medical institutions for medical treatment. If local medical institutions still cannot provide services, they will be remotely guided by the Affiliated Hospital of Chifeng university or transferred to the Affiliated Hospital of Chifeng university through green channel. Based on this, it is more convenient for health service objects to seek medical treatment, and it can also promote medical institutions at all levels to join the service system. The joined social medical resources become local sub centers. All data generated by the sub centers shall be summarized to the Affiliated Hospital of Chifeng University, and the data shall be uploaded and unified in real time.

\section{Service steps of health management in the Affiliated Hospital of Chifeng University}

Health management refers to the whole process of comprehensive monitoring, evaluation, analysis and intervention of individual health risk factors, and providing health consultation and guidance. It includes health management services such as health status information collection, health status evaluation and prediction, health intervention and consultation and guidance. It is a closed-loop service process (Figure 2).
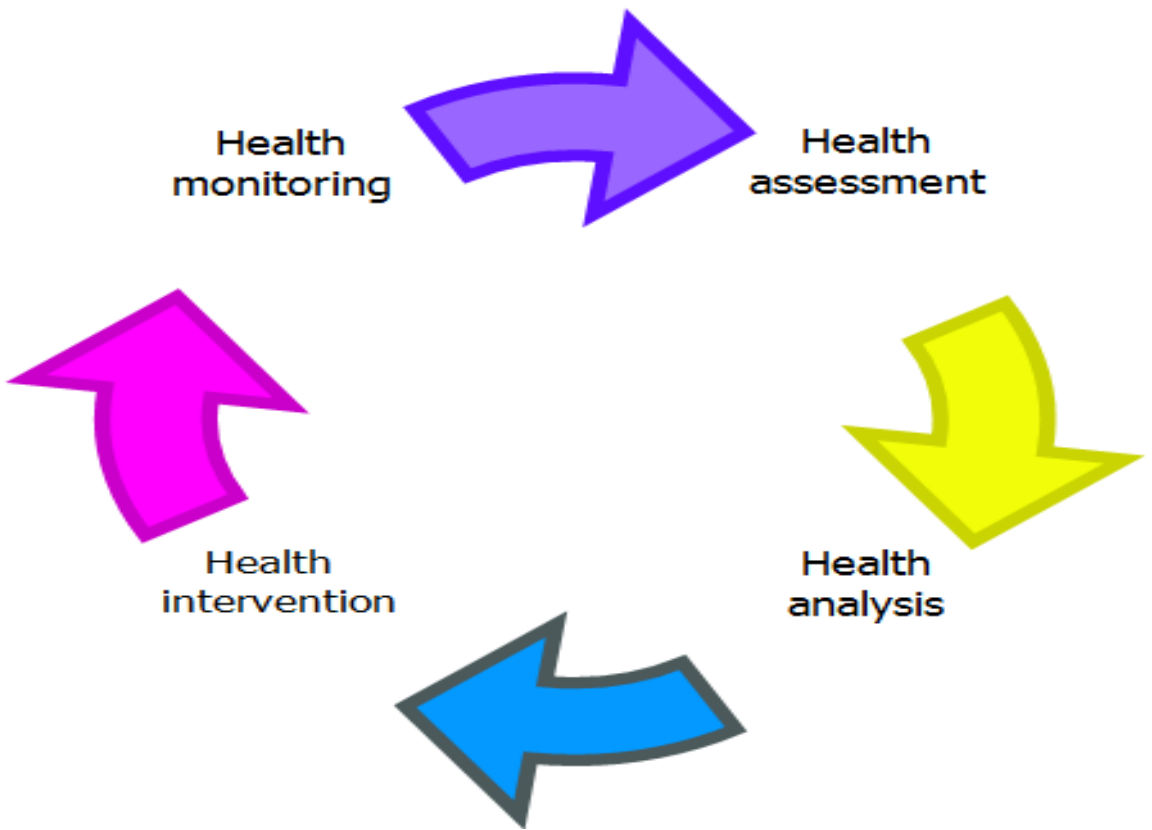

Figure 2. Closed loop flow chart of health management service

\subsection{Health information collection}

Health information collection is the basis of health management. Whether the information collection is comprehensive and accurate is the basis of the success of health management. Health management information comes from: first, basic information (such as blood pressure and exercise) comes from the 
health monitoring system worn outside the hospital; Second, it comes from the physical examination database; Third, through regular monitoring and interactive collection of information into the database; Fourth, from the community or other physical examination institutions. The above information will be uploaded to the information center of the health examination hospital in real time.

\subsection{Health monitoring}

Health monitoring is the process of discovering health risk factors. The system screens the personal health information of the health management object, finds out the regular information, uploads the abnormal data in real time and gives an automatic alarm, or the health manager monitors and finds the risk factors according to the health information, prompts and gives early warning to the health indicators beyond the normal range, or provides the monitoring data analysis report

\subsection{Health status evaluation and prediction}

Health status evaluation and prediction is the process of understanding health risk factors. Accurate and clear characteristics of health services and disease prevention strategies must be achieved through health status evaluation and prediction. The health manager will analyze the abnormal data according to the basic information, physical examination information, regular monitoring and interactive information of the health management object, and make a one-to-one and personalized evaluation. If the health manager is unable to provide health services to the health object, it is necessary to ask the health manager of the local and superior health management organization to make evaluation and prediction according to the operation mechanism formulated by the health service operation organization.

\subsection{Health intervention}

Health promotion behavior intervention and counseling are the process of solving health risk factors, which also reflects the consistency of health care. Health managers follow the health service standards formulated by the health examination hospital, obtain health information in real time, conduct health assessment according to the health knowledge base or standards of the health examination hospital, and carry out health intervention and consultation guidance through health guidance and return visit. Once the complications caused by health risk factors are found, they shall be immediately admitted to the hospital for special disease management (when entering the medical service link, the doctor shall monitor, evaluate and analyze the results of the indicators of the health service objects through the health manager according to the medical service standards, and carry out the adjustment of treatment scheme, drugs and examination frequency in the medical link). For follow-up treatment problems such as health risk management after discharge, the health manager will continue to track their health status, and provide appointment tracking service, regular observation and re-examination, green channel admission and other services.

\section{Summary}

This paper discusses the establishment of the health management service model of the Affiliated Hospital of Chifeng University, the establishment of a health examination hospital, the mobilization of social resources, joint participation, division of labor and cooperation. The Affiliated Hospital of Chifeng university provides medical and technical support, and the health examination hospital guides and supports social resources to develop health management services in the region. To adopt network management, we need to establish a unified system, mechanism, system and management mode to realize integrated management. Only with the participation of the Affiliated Hospital of Chifeng University and other Class $3 \mathrm{~A}$ hospitals, and as the engine of health management services, driving health management services, can the integrity and sustainability of the whole process of health in Chifeng area be realized. 


\section{Funding}

The 2021 Chifeng natural science research project "Research on Optimization of health management mode of Chifeng Class 3A general public hospital” (Project number: 2021-40)

\section{Disclosure statement}

The author declares no conflict of interest.

\section{References}

[1] Loeppke R, 2010, Edington DW, BEGS. Impact of the Prevention Plan on Employee Health Risk Reduction. Population Health Management, 13(5): 275- 284.

[2] Nash DB, 2011, Population Health Management in Medicare: The Time is Now. Population Health Manage, 14(1): S1.

[3] Loza CL, Castillo-Portilla M, Rojas JL, et al., 2011, Basic Principles and Methodological Considerations of Health Economic Evaluations. Rev Peru Med ExpSalud Publica, 28(3): 518-527.

[4] McCarver P, 2011, Success of a Diabetes Health Management Program in Employer Based Health Care Centers. AAOHN J, 59(12):513-518.

[5] Pirkko V, 2008, Health Care Management in Finland: An Analysis of the Wickedness of Selected Reforms. Review of Business, 28(2):41-55. 